\title{
TEORIA DE OPÇÕES \\ APLICADA A PROJETOS DE INVESTIMENTO
}

Andrea Maria Accioly Fonseca Minardi

Professora do IBMEC Business School e Doutoranda em Administração de Empresas na EAESP/FGV.

E-mail: minardi@ibmec.br

\section{RESUMO}

A teoria de opções é a melhor abordagem para integrar estratégia e finanças, pois considera, analiticamente, as opções de crescimento e as flexibilidades gerenciais, ou as formas de adaptação às mudanças do ambiente empresarial, que são o cerne da estratégia empresarial. Um projeto pode ser considerado como uma série de opções reais, como, por exemplo, postergar o investimento, contrair ou expandir a escala de produção, abandonar temporária ou definitivamente o projeto e investir em projetos dependentes deste primeiro. As opções reais são avaliadas de maneira análoga às opções financeiras.

\section{ABSTRACT}

Option theory is the best approach to integrate finance and strategy. It quantifies growth options and management flexibility, that are the heart of business strategy. A project can be viewed as a set of real options, as defer the investment, expand or contract operational scale, abandon, temporarily or definitively, investments in subsequent projects, switching inputs and outputs. Real options can be precified in a way analogous to financial options.

PALAVRAS-CHAVE: Opções reais, teoria de opções, flexibilidade gerencial, integração entre estratégia e finanças.

KEY WORDS: Real option, option pricing theory, management flexibility, integration between strategy and finance. 


\section{INTRODUÇÃO}

Hoje em dia, qualquer indústria pode estar sujeita a fenômenos como globalização, inovação tecnológica constante e desregulamentação. O cenário empresarial atual é altamente competitivo e incerto, e algumas indústrias podem estar se transformando drasticamente, com novas formas emergentes de relacionamento entre clientes e fornecedores e novos competidores, antes inimagináveis. Cada vez mais, é vital gerenciar empresas de uma maneira flexível e rever constantemente estratégias e planos antes concebidos. A adaptação rápida e mesmo a antecipação às mudanças do ambiente empresarial são imprescindíveis para a sobrevivência da empresa em longo prazo.

As tomadas de decisões realizadas hoje influenciam o futuro da empresa. Infelizmente, as técnicas quantitativas de avaliação de projetos, sendo a principal delas o fluxo de caixa descontado ou o método do valor presente líquido, nem sempre conduzem à melhor solução estratégica. Os executivos estão sujeitos a tomar decisões erradas baseando-se tanto em análises quantitativas quanto unicamente em sua intuição.

Podemos dizer que a flexibilidade gerencial ou as formas de adaptação às mudanças do ambiente empresarial nada mais são que uma série de opções reais, algumas inerentes aos projetos e outras construídas mediante um custo maior de investimento como:

a) postergar um projeto: o investimento em um projeto pode ser postergado para se obterem melhores informações sobre o mercado, resolvendo algumas incertezas. No caso de o mercado indicar um desempenho pior que o inicialmente previsto, o desembolso com o investimento será poupado.

b) expandir ou contrair a escala de produção: se as condições do mercado se tornarem melhores que as previstas, é possível ampliar a escala de produção mediante um investimento adicional, aumentandose o ganho do projeto. Caso as condições do mercado se tornem piores que as previstas, é possível diminuir a escala de produção, limitando-se as perdas do projeto.

c) abandonar temporária ou definitivamente um projeto: caso as condições de mercado se tornem muito desfavoráveis ao projeto, é possível abandonálo temporariamente, na espera de melhores condições de mercado para retomar o projeto ou até mesmo abandoná-lo por completo.

d) abandonar o projeto ainda em fase de construção: na maior parte das vezes, o desembolso de um projeto não ocorre todo de uma só vez, mas à medi- da que o projeto vai sendo construído. Caso as condições de mercado se tornem muito piores do que o inicialmente previsto, é possível abandonar as outras etapas da construção do projeto, poupando parcelas do investimento previsto.

e) alterar as matérias-primas ou os produtos finais de um projeto: um processo mais flexível permite que se altere o uso de matérias-primas conforme as condições do mercado se modificam. Por exemplo, um boiler que pode utilizar tanto gás como energia 
expectativas iniciais. Ao ignorar as opções reais existentes, o método do valor presente líquido subavalia projetos e pode levar a uma tomada de decisão errada, como, por exemplo, rejeitar projetos que seriam estrategicamente interessantes para as empresas ou aceitar processos mais rígidos em detrimento de processos mais flexíveis, podendo comprometer a posição estratégica futura da empresa.

c) Simulação de Hertz (1964): a técnica procura considerar todas as combinações possíveis das variáveis, considerando as inter-relações existentes e dando como resultado uma distribuição de probabilidades de fluxos de caixa para cada período ou de valores presentes líquidos para o projeto. O problema mais difícil em uma simulação consiste em estimar a interdependência entre as variáveis e a distribuição de probabilidades subjacentes.

A teoria de opções é a melhor abordagem para avaliar projetos que possuem opções operacionais e estratégicas significativas, conseguindo integrar estratégia e finanças.

O valor das opções depende do valor de outro ativo, que é denominado ativoobjeto. O projeto é visto como um conjunto de opções reais, que tem como ati-

O valor presente líquido pode ser aplicado sem problemas quando as flexibilidades gerenciais não são significativas. Caso contrário, precisa ser remodelado para capturar o valor dessas flexibilidades.

$V_{\text {PLexpandido }}=$ VPL tradicional + Valorflexibilidade gerencial

\section{TÉCNICAS DE AVALIAÇÃO QUE CAPTURAM O VALOR DE FLEXIBILIDADES GERENCIAIS}

Para corrigir as deficiências do VPL em capturar o valor de flexibilidade gerencial, surgiram alguns modelos alternativos, tais como:

a) Análise de cenários: o projeto é analisado sob a óptica de vários cenários. Para cada cenário, é atribuída uma probabilidade, e o valor do projeto é a média ponderada dos diversos cenários. Embora esse modelo seja bastante utilizado na prática, é impossível prever todos os cenários possíveis, e a probabilidade considerada de ocorrência de cada cenário é totalmente subjetiva.

b) Árvore de decisão de Magee (1964): procura antecipar as ações gerenciais que ocorrerão em virtude das mudanças das condições de mercado. O valor presente líquido é função não apenas das condições de mercado esperadas no início do projeto mas também das futuras ações gerenciais. A cada momento, cada ramo da árvore corresponde a uma decisão, baseada em uma situação de mercado. A probabilidade atribuída a cada ramo é subjetiva, tendo cada ramo um risco diferente e, portanto, devendo ter uma taxa de desconto diferente, o que torna a implementação à risca do modelo bastante complexa. vo-objeto o valor do projeto. Essas opções reais são avaliadas de uma maneira análoga às opções financeiras.

A teoria de opções tenta superar as limitações dos métodos tradicionais, pois considera as flexibilidades gerenciais na avaliação e utiliza como taxa de desconto a taxa de retorno do ativo livre de risco, não se preocupando em determinar uma taxa de desconto de acordo com o risco do projeto.

Independentemente da preferência e percepção de risco, todos os investidores concordarão com uma mesma relação entre preço de opção, preço do ativoobjeto, volatilidade (desvio-padrão das taxas de retorno do ativo-objeto), tempo até o vencimento da opção e dividendos ou efeito dividendos existentes durante a vida da opção. Essa concordância implica que opções são avaliadas em um mundo neutro ao risco, e isso permite que se utilize a taxa de retorno do ativo livre de risco.

A subjetividade da teoria de opções é bastante reduzida em relação às outras técnicas, mas não é totalmente eliminada. $\mathrm{O}$ valor das opções é dependente do valor do projeto. O comportamento do valor do projeto é considerado como uma variável estocástica, isto é, que tem um comportamento aleatório e regido por alguma distribuição de probabilidade, normalmente a distribuição normal ou a lognormal. A variável-chave da distribuição de probabilidade é a volatilidade do comportamento do ativo-objeto, ou seja, o desvio-padrão das taxas de retorno do projeto.

A volatilidade do projeto pode ser obtida de duas maneiras:

a) histórica: por meio de uma série histórica; nesse caso, assume-se que a volatilidade futura do projeto será a mesma que a volatilidade passada. 
b) implícita: quando existem opções sobre o ativoobjeto comercializadas no mercado, é possível determinar sua volatilidade implícita por meio do modelo Black-Scholes. Como existe normalmente mais de uma opção emitida sobre um mesmo ativoobjeto, será encontrada mais de uma volatilidade, sendo necessário utilizar alguma forma de ponderação para se obter uma média entre as diversas volatilidades. Essa ponderação é sempre subjetiva.

\section{ANALOGIA ENTRE OPÇÕES REAIS E OPÇÕES FINANCEIRAS}

Basicamente, as opções reais podem ser precificadas de maneira análoga às opções financeiras. $\mathrm{O}$ Quadro 1 resume como pode ser feita a analogia entre opções reais e opções financeiras.

Grande parte das opções financeiras pode ser avaliada pelo modelo Black-Scholes. No entanto, opções reais são muito mais complexas.
As principais diferenças entre opções reais e financeiras são:

a) Opções financeiras típicas têm vida curta (menos de 1 ano para expiração, embora existam algumas warrants de 5 a 10 anos). Opções reais têm vida longa, sendo perpétuas em alguns casos.

b) Embora opções financeiras possam ser exercidas antecipadamente (antes do vencimento), a análise desse exercício é secundária, pois, dada a vida curta da opção, o custo de não a exercê-la no momento ótimo não é significativo. Em opções reais, no entanto, a decisão de exercer antecipadamente uma opção ou de se adotar um projeto é crítica.

c) Os ativos-objetos de opções financeiras são comercializados em vários mercados e, como seus preços nunca serão negativos, a escolha dos processos aleatórios para a precificação do ativoobjeto é mais limitada.

Em opções reais, o ativo-objeto (projeto) geralmente não é comercializado e nada garante que seu va-

\section{Quadro 1 - Analogia entre opções reais e opções financeiras}

\begin{tabular}{|c|c|}
\hline Postergar & $\begin{array}{l}\text { Opção de compra americana do valor presente do projeto } \\
\text { ( } V=\text { preço do ativo-objeto), mediante desembolso do investimento ( } I=\text { preço de exercício) } \\
V P L_{\text {expandido }}=\operatorname{máx}(V-I, 0)\end{array}$ \\
\hline $\begin{array}{l}\text { Cancelar novas } \\
\text { etapas do } \\
\text { investimento }\end{array}$ & $\begin{array}{l}\text { Opção composta em que cada etapa da construção é encarada como uma opção de } \\
\text { compra no valor das etapas subseqüentes, sendo o preço de exercício a prestação do } \\
\text { investimento necessária para prosseguir para a próxima etapa }\end{array}$ \\
\hline $\begin{array}{l}\text { Expandir a } \\
\text { escala de } \\
\text { produção }\end{array}$ & $\begin{array}{l}\text { Opção de compra de uma parcela de } x \% \text { da escala-base do projeto } \\
(x V=\text { preço do ativo-objeto }) \text {, mediante o investimento adicional }(l e=\text { preço de exercício }) \\
V P_{\text {expandido }}=V+\operatorname{máx}(x V-l e)\end{array}$ \\
\hline $\begin{array}{l}\text { Contrair a } \\
\text { escala de } \\
\text { produção }\end{array}$ & $\begin{array}{l}\text { Opção de venda de uma parcela de } c \% \text { da escala-base do projeto } \\
\text { ( } c V=\text { preço do ativo-objeto), economizando uma parte dos custos }(I c=\text { preço de exercício) } \\
V P_{\text {expandido }}=V+\operatorname{máx}(I c-c V, 0)\end{array}$ \\
\hline $\begin{array}{l}\text { Fechar } \\
\text { temporariamente }\end{array}$ & $\begin{array}{l}\text { A operação de cada ano é vista como uma opção de compra de receita de caixa do ano } \\
(R=\text { preço do ativo-objeto), tendo como preço de exercício os custos operacionais } \\
\text { variáveis }(I V=\text { preço de exercício) } \\
V_{\text {opcaão }=m a ́ x(R-I V, 0)} \\
\text { Podemos também encarar o problema como uma opção entre obter o valor do projeto } \\
V \text { (líquido dos custos fixos) menos os custos variáveis } I V \text { e fechar e receber o valor do } \\
\text { projeto menos a receita de caixa prevista para o ano, } R \\
V P_{\text {expandido }=\text { máx }(V-I V, V-R)-I F=(V-I F)-m i ́ n(I V, R)}\end{array}$ \\
\hline $\begin{array}{l}\text { Abandonar } \\
\text { pelo valor }\end{array}$ & $\begin{array}{l}\text { Opção de venda americana do valor do projeto } \\
(V=\text { preço residual do ativo-objeto), recebendo-se o valor residual }(A=\text { preço de exercício) } \\
V_{\text {opcão }}=\operatorname{máx}(A-V, 0) \\
V P_{\text {expandido }}=\operatorname{máx}(A, V)\end{array}$ \\
\hline
\end{tabular}


lor não se torne negativo, o que pode implicar trabalhar-se com uma maior gama de processos aleatórios para o valor do ativo-objeto. Além disso, se não for possível encontrar um ativo negociado no mercado perfeitamente correlacionado com o projeto analisado, a avaliação da teoria de opções perde sua acurácia, uma vez que a volatilidade do projeto é estimada subjetivamente, sem uma base fundamentada.

d) Opções financeiras têm um preço de exercício determinado e único. O preço de exercício de opções reais, geralmente o valor do investimento no projeto, pode variar ao longo do tempo, podendo até variar de maneira aleatória.

e) Opções financeiras geralmente envolvem uma única opção. Opções reais geralmente são compostas. Por exemplo, o setor de P\&D cria uma opção para se adotar uma tecnologia com benefícios desconhecidos. Se a tecnologia desenvolvida for bemsucedida, existe uma opção para expandir a linha de produto se houver sucesso no mercado final. Se o produto, no entanto, se tornar obsoleto, existe a opção de abandonar sua produção. Uma análise adequada de $\mathrm{P} \& \mathrm{D}$ deve incluir o valor de opções subseqüentes de expansão e abandono. Infelizmente, o valor de opções múltiplas é diferente da soma dos valores individuais das opções, porque opções reais interagem entre si. Por exemplo, uma vez abandonado o projeto, não haverá mais a opção de contrair a escala de produção. O modelo binomial logarítmico transformado de Trigeorgis (1991) é indicado para se avaliar opções múltiplas.

f) Opções financeiras são exclusivas do investidor. Nenhuma outra pessoa pode exercer a opção possuída por ele. O mesmo não ocorre com opções reais, em que o grau de exclusividade depende da estrutura de mercado. Se o mercado for monopolista, a empresa tem a exclusividade total de investimento em seu setor. Se o mercado for perfeitamente competitivo, o direito de investimento é um bem público de toda indústria, e o valor da opção real se deteriora com o passar do tempo. Por exemplo, o setor de P\&D de uma determinada empresa desenvolveu um produto inovador. $\mathrm{O}$ valor de se lançar esse produto hoje pode ser maior que o valor de se lançar esse produto amanhã, pois outras empresas concorrentes poderão desenvolver produtos similares. Neste caso, o valor da opção de postergar o lançamento deteriora-se com o tempo.

g) A eficiência do mercado financeiro ajusta os preços rapidamente, refletindo todas as informações correntes. Retornos acima do mercado não são duradouros e o que prevalece é a taxa de equilíbrio.
No entanto, mercado de bens e serviços são geralmente menos que perfeitamente eficientes, permitindo a uma empresa ter ganhos acima do equilíbrio de mercado enquanto perdurar sua vantagem competitiva.

\section{UM EXEMPLO NUMÉRICO DA APLICAÇÃO DA TEORIA DE OPÇÕES PARA AVALIAR PROJETOS}

Uma empresa de mineração está pensando em investir em uma jazida de cobre, necessitando para isso desembolsar \$ 104 milhões. O valor presente atual de se explorar a jazida hoje é \$ 100 milhões (perfeitamente correlacionado com o preço do cobre) e, historicamente, o preço do cobre possui uma volatilidade de $58,78 \%$ a.a.

Se não houver opções reais significativas, o valor presente líquido do projeto é - $\$ 4$ milhões (\$100 milhões - \$ 104 milhões).

Consideremos agora que exista a possibilidade de abandonar definitivamente a mina por um valor residual de $\$ 70$ milhões (melhor uso do terreno), caso o preço do cobre se torne muito baixo.

A flexibilidade gerencial de abandonar a mina cria uma assimetria ou inclinação na distribuição de probabilidade do VPL do projeto.

Para capturar o valor da opção de abandono, o VPL precisa ser remodelado para:

$$
\text { VPLexpandido }=\text { VPLtradicional }+ \text { Valor }_{\text {opção de abandono }}
$$

A opção de abandono é análoga a uma opção de venda do valor presente do projeto $(V)$, em que o preço de exercício é igual ao valor residual $(A)$. O payoff da opção de abandono é igual a máx $(A-V, 0)$.

Sabendo-se que a taxa do ativo livre de risco é $10 \%$ a.a. e aplicando-se a equação de Black-Scholes para opção de venda, obtemos:

Opção abandono $=70 \mathrm{e}^{-0,1} N(-d 2)-100 N(-d 1)$
$d 1=\frac{\ln \left(\frac{100}{70}\right)+\left(0,1+\frac{0,5878^{2}}{2}\right)}{0,5878}=1,0708$
$d 2=\frac{\ln \left(\frac{100}{70}\right)+\left(0,1-\frac{0,5878^{2}}{2}\right)}{0,5878}=0,4830$
$\begin{gathered}\text { Opção de abandono }=\$ 5,76 \text { milhões } \\ \text { Valor presente líquido expandido }=-\$ 4 \text { milhões }+\$ 5,76 \\ \text { milhões }=\$ 1,76 \text { milhão }\end{gathered}$




\section{CONCLUSÃO}

A teoria de opções, quando aplicada a projetos de investimento, integra estratégia e finanças, pois considera, analiticamente, as flexibilidades gerenciais e as opções de crescimento, que são o cerne da estratégia empresarial. O método do VPL subavalia projetos que possuem opções reais significativas. A avaliação com o uso da teoria de opções, por outro lado, pressupõe que será exercida sempre a política ótima de investimento, o que nem sempre é possível, podendo resultar em estimativas de valores mais altos do que os reais. No entanto, fornece um parâmetro de valor bem melhor que o apurado pelo VPL, resultando em uma boa base para iniciar negociações.

Copeland, Koller e Murrin (1994) citam um caso prático no qual a McKinsey Co. foi incumbida de avaliar uma mineradora produtora de um determinado minério, responsável por um terço da oferta mundial. As estimativas iniciais do valor presente líquido da mineradora, baseadas nas previsões de preço do minério, atingiram apenas $45 \%$ do valor de mercado do patrimônio líquido da empresa. Uma análise de cenários elevou esse valor para $71 \%$ do valor de mercado. A avaliação por meio da teoria de opções elevou esse valor para $116 \%$ do valor corrente da empresa no mercado. As opções de fechamento, reabertura e abandono equivaleram de $5 \%$ a $15 \%$ do valor da área de produção correspondente, considerando um preço para o minério na faixa de US\$1,75/onça a US\$2,25/ onça. O valor dessas opções era muito maior para preços mais baixos do minério e muito menor para preços do minério mais altos.
Uma outra vantagem da teoria de opções consiste em, estabelecendo uma política ótima operacional, conscientizar a empresa sobre qual seria o melhor momento de agir (por exemplo, abandonar um projeto, fechar temporariamente para reabrir mais tarde, expandir ou contrair a produção, etc.), tornando a gerência da empresa mais perto da ótima.

Basicamente, a teoria de opções pode ser aplicada a projetos de investimento não comercializados no mercado, desde que exista no mercado uma carteira que esteja altamente correlacionada com o projeto. A impossibilidade de se montar essa carteira diminui bastante a acurácia da avaliação, que se torna, na melhor das hipóteses, uma avaliação grosseira.

Opções reais podem ser bastante complexas e, embora as abordagens que aproximam as equações diferenciais parciais resultem em avaliações mais acuradas, abordagens que aproximam diretamente o processo estocástico, como o modelo binomial, são bastante viáveis para fins práticos, com a vantagem de serem bastante intuitivas.

Opções reais existentes em um único projeto de investimento geralmente não podem ser somadas, pois interagem. O modelo binomial logarítmico transformado de Trigeorgis consiste em uma ferramenta interessante para avaliar um projeto com mais de uma opção.

A área em que a teoria de opções é mais largamente utilizada consiste na área de recursos naturais, pois os preços dos ativos-objetos podem ser obtidos diretamente do mercado. A teoria também tem sido utilizada em áreas como $\mathrm{P} \& \mathrm{D}$, avaliação de terrenos, estratégia internacional, flexibilidade de alternar matéria-prima e produtos finais. $\bigcirc$

REFERÊNCIAS BIBLIOGRÁFICAS

COPELAND, T., KOLLER, T., MURRIN, J. Valuation: measuring and managing the value of companies. New York : John Wiley \& Sons, 1994.
HERTZ, D. Risk analysis in capital investment. Harvard Business Review, p. 95-106, Jan./Feb. 1964.

MAGEE, J. F. How to use decision trees in capital investments. Harvard Business Review, p. 126-8, Sept./Oct. 1964.
TRIGEORGIS, L. A log-transformed binomial numerical analysis method for valuing complex multi-option investments. Journal of Financial and Quantitative Analysis, v. 26, n. 3, p. 309-26, Sept. 1991.
BIBLIOGRAFIA ADICIONAL

Existem inúmeros bons textos que tratam do assunto opções reais. Podemos, entre outros, recomendar:

ANKUM, L., SMIT, Han T. Real options and game theoretic approach to corporate investment strategy under competition. Financial Management, v. 22, n. 3, p. 241-50, Autumn 1993.

BRENNAN, M., SCHWARTZ, E. Evaluating natural resource investments. Journal of Business, v. 58, n. 2, p.135-157, Apr. 1985.

BRENNAN, M., TRIGEORGIS, L. Project flexibility, agency and competition: new developments in the theory and application of real options. New York : Oxford University Press, 2000.
DIXIT, A., PINDYCK, R. Investment under uncertainty. Princeton NJ : Princeton University Press, 1994.

KUTILAKA, N. The value of flexibility: the case of a dual fuel industrial steam boiler. Financial Management, v. 22, n. 3 , p. 271-280, Autumn 1993.

MYERS, Stewar C. Finance theory and financial strategy. Midland Corporate Finance Journal, New York, n. 5, p. 6-13, Spring 1987.

SICK, G. Capital budgeting with real option. New York Salomon Brothers Center for the Study of Financial Institution, 1989. (Monograph Series in Finance and Economics Monograph 1989 -3).
TRIGEORGIS, L. Real option and interaction with financial flexibility. Financial Management, v. 22, n. 3, p. 202-24, Autumn 1993.

TRIGEORGIS, L. The nature of option interactions and the valuation of investments with multiple real option. Journal of Finance and Quantitative Analysis, v. 28, n.1, p. 1-20, Mar. 1993.

TRIGEORGIS, L. Real options in capital investment models, strategies and applications. Westport, Connecticut : Praeger, 1995.

TRIGEORGIS, L. Real options: managerial flexibility and strategy in resource allocation. Cambridge, Mass : MIT Press, 1996. 